\title{
Entanglement and entropy operator for strings in pp-wave time dependent background
}

\author{
A. L. Gadelha*, Dáfni Z. Marchioro ${ }^{\dagger}$ and Daniel L. Nedel ${ }^{\ddagger}$ \\ Instituto de Física Teórica, UNESP - São Paulo State University, \\ Rua Pamplona 145, São Paulo, SP, 01405-900, Brazil
}

\begin{abstract}
In this letter new aspects of string theory propagating in a pp-wave time dependent background with a null singularity are explored. It is shown the appearance of a $2 \mathrm{~d}$ entanglement entropy dynamically generated by the background. For asymptotically flat observers, the vacuum close to the singularity is unitarily inequivalent to the vacuum at $\tau=-\infty$ and it is shown that the $2 \mathrm{~d}$ entanglement entropy diverges close to this point. As a consequence, the positive time region is inaccessible for observers in $\tau=-\infty$. For a stationary measure, the vacuum at finite time is seen by those observers as a thermal state and the information loss is encoded as a heat bath of string states.
\end{abstract}

\footnotetext{
* gadelha@ift.unesp.br

$\dagger$ dafni@ift.unesp.br

$\ddagger$ daniel@ift.unesp.br
} 


\section{INTRODUCTION}

One of the most interesting subjects in string theory is its study in time dependent backgrounds. Such endeavour may provide answers to important questions involving typical quantum cosmology problems from the string theory point of view, such as the initial conditions, the cosmological singularities and the pre-big bang scenario.

In general, string theory in time dependent backgrounds is hard and demands further development of its non perturbative sector. On the other hand, string theory in time dependent plane wave backgrounds is always in the perturbative regime and it is exactly solvable, providing insights in many aspects of the theory in more complicated geometries ${ }^{1}$. In particular, in [5] the string theory is quantized in a pure dilatonic singular plane wave metric, where the worldsheet model exhibits many interesting properties (or troubles) of field theory in curved space, including the choice of the vacuum and particle creation (in this case string mode creation). In the Brinkmann coordinates the metric is asymptotically flat and defined in the interval $-\infty \leq \tau \leq+\infty$ of the light-cone time, with a null singularity at the origin $(\tau=0)$. So the model shares many characteristics with the Gasperini and Veneziano's pre-big bang cosmology [6], and it can be used as a toy model to investigate this scenario. ${ }^{2}$

In this work we analyze the results of [5] in Brinkmann coordinates to explore different aspects of time dependent backgrounds in the worldsheet context: the study of entanglement entropy and loss of information. Concerning closed string propagating in a pp-wave time dependent background, with asymptotically flat regions $(\tau= \pm \infty)$, it was shown in [5] (and references therein) that there is creation of string modes, when the string propagates from one of the asymptotically flat regions to the singularity in $\tau=0$, as seen by an observer in the flat vacuum $\left|0(\tau= \pm \infty), p_{v}\right\rangle$. This result is of course observer dependent. In this letter we show that the interaction of the string with the time dependent background, as seen by a flat observer, creates a state with a particular structure of excitation, characterized by a condensation of right and left moving string modes. This particular structure in a finite $\tau$ is the core of the development we intend to do, presenting a scenario where the entanglement generated by the background, the entanglement entropy, loss of information and macroscopic effects (thermal) can be studied.

\footnotetext{
${ }^{1}$ For a historical development of the subject see, for example, 1, 2, 3, 4].

2 See also the Maurizio Gasperini's home page http://www.ba.infn.it/ gasperin/
} 
The establishment that the vacuum state at finite $\tau$ is a condensed state is possible due to the observation that there is a Bogoliubov transformation mapping the asymptotically flat string Hilbert space to its corresponding one at a finite $\tau$. This Bogoliubov transformation is formally identical to the general unitary SU(1,1) Thermo Field Dynamic (TFD) transformation, proposed and analyzed in [7, 8, 9] $]^{3}$. However, this identification is just formal. In TFD, there is a duplication of the system's degrees of freedom by means of introducing an auxiliary system (copy of the original (physical) one), followed by a Bogoliubov transformation that entangles elements of the two subspaces (original and auxiliary) ${ }^{4}$. In the case we are studying, there is no duplication of the system's degrees of freedom and the Bogoliubov transformation entangles the left and right moving string modes in such a way that our condensed state is in fact an entangled state of these modes.

It is known that in TFD the condensed state can be generated by an entropy operator. This operator can be interpreted as an entanglement entropy operator and has been used in different physical situations, where TFD-like condensed states appear [12, 13, 14, 15, 16]. As we also have a condensed state with a TFD structure, we can look for an entropy operator which generates this state and explore the physical consequences of this fact. Actually we show that the entropy operator appears in a different context: it represents a $2 d$ entanglement entropy, dynamically generated by the time dependent background. This comes from the fact that, although this time dependent geometry does not produce string creation, from the worldsheet point of view, the metric produces creation of string modes and all the information about the background is contained in the entropy operator.

We analyze the entropy operator expectation value at the singularity and we show that it diverges owing to the infinite creation of string modes. Even when the singularity is avoided by an analytic continuation, the behaviour remains the same. Furthermore, we show that the string's Hilbert space close to the singularity is non unitarily related to the flat Hilbert space, defined in $\tau=-\infty$. This is also a result of the infinite creation of string modes close to the singularity. In summary, although the singularity can be avoided by an analytical continuation of the Bessel functions, for observers defined in $\tau=-\infty$, there is a complete loss of information about the string states at the singularity and the positive time region is

\footnotetext{
${ }^{3}$ Recently in [10] a general unitary $S U(2)$ TFD formulation was also proposed to study type IIB GreenSchwarz superstring at finite temperature.

4 Recently it was shown that TFD states, seen as pure states, are maximum entangled states [1].
} 
inaccessible.

Finally, we look for macroscopic effects related to the generation of entropy and the respective information loss. We show that in a particular (stationary) situation the expectation value of the flat number operator in the vacuum at finite time acquires the form of a Boltzmann distribution by an appropriate choice of parameters. This result is interpreted as a heat bath perceived by the asymptotically flat observer when the string evolves in the pp-wave time-dependent background.

The letter is divided as follows: in section two, we review the model and fix the notation; in sections three and four we present our results, and section five is devoted to conclusion and discussion of the results.

\section{THE MODEL}

In this section, we present a review of the results obtained in [5] for the model we want to analyze ${ }^{5}$. We are going to consider type IIB Green-Schwarz superstring in the following pp-wave time-dependent background (in Brinkmann coordinates)

$$
\begin{array}{r}
d s^{2}=2 d u d v-\lambda x^{2} d u^{2}+d x^{i} d x^{i}, \\
\phi=\phi(u), \quad \lambda=\frac{k}{u^{2}},
\end{array}
$$

where $u, v$ are the light-cone coordinates, $x^{i}(i=1 \ldots d)$ are the transverse coordinates and $\phi$ is the dilaton. This metric has a null singularity at $u=0$. Choosing the light-cone gauge to fix the reparametrization invariance,

$$
u=2 \alpha^{\prime} p^{u} \tau
$$

the bosonic part of the action is

$$
S=-\frac{1}{4 \pi \alpha^{\prime}} \int d \tau \int_{0}^{\pi} d \sigma\left(\partial^{a} x^{i} \partial_{a} x^{j} \delta_{i j}+\frac{k}{\tau^{2}}\left(x^{i}\right)^{2}\right)
$$

and the equations of motion for the transverse coordinates read

$$
\left(\partial_{\tau}^{2}-\partial_{\sigma}^{2}\right) x^{i}+\frac{k}{\tau^{2}} x^{i}=0
$$

\footnotetext{
5 The time dependent pp-wave background was also recently studied in the presence of a Ramond-Ramond 5 -form field strength [17]. See also [18, 19].
} 
If we perform a change of coordinates from Brinkmann to Rosen,

$$
\begin{gathered}
v=V+\frac{1}{2} h(u) X^{i} X^{i}, \quad x^{i}=a(u) X^{i}, \\
h=-a \frac{d a(u)}{d u}, \quad \frac{d^{2} a(u)}{d u^{2}}=-\lambda(u) a(u),
\end{gathered}
$$

the metric takes the form

$$
\begin{array}{r}
d s^{2}=2 d u d V+a^{2}(u) d X^{i} d X^{i}, \\
a(u)=u^{1-\nu}, \quad \nu=\frac{1}{2}(1+\sqrt{1-4 k}), \quad 0<k<\frac{1}{4}
\end{array}
$$

and the equations of motion for the transverse coordinates $X^{i}$ resemble the equation of a damped harmonic oscillator [5],

$$
\left(\partial_{\tau}^{2}+\frac{\gamma}{\tau} \partial_{\tau}-\partial_{\sigma}^{2}\right) X^{i}=0, \quad \gamma=2(1-\nu)
$$

with friction coefficient proportional to $\frac{\gamma}{\tau_{0}}$ in a small region of time around some $\tau_{0}$. So, in Rosen coordinates it is clear that this metric produces dissipation in the $2 \mathrm{~d}$ worldsheet field theory. As the entropy production and loss of information appear naturally in some dissipative systems [14, 15], the Rosen coordinate system may be the natural one to make the analysis we intend to do. However, we are going to work in Brinkmann coordinates because it is asymptotically flat, which makes the analysis easier.

The solution of the equations of motion in Brinkmann coordinates can be written as

$$
\begin{aligned}
x^{i}(\sigma, \tau) & =x_{0}^{i}(\tau)+\frac{1}{2} \sqrt{2 \alpha^{\prime}} \sum_{n=1}^{\infty} \frac{1}{\sqrt{n}}\left[Z(2 n \tau)\left(a_{n}^{i} e^{2 i n \sigma}+\tilde{a}_{n}^{i} e^{-2 i n \sigma}\right)\right. \\
& \left.+Z^{*}(2 n \tau)\left(a_{n}^{i \dagger} e^{-2 i n \sigma}+\tilde{a}_{n}^{i \dagger} e^{2 i n \sigma}\right)\right]
\end{aligned}
$$

with

$$
\begin{array}{r}
Z(2 n \tau) \equiv e^{-i \frac{\pi}{2} \nu} \sqrt{\pi n \tau}\left[J_{\nu-\frac{1}{2}}(2 n \tau)-i Y_{\nu-\frac{1}{2}}(2 n \tau)\right] \\
x_{0}^{i}(\tau)=\frac{1}{\sqrt{2 \nu-1}}\left(\tilde{x}^{i} \tau^{1-\nu}+2 \alpha^{\prime} \tilde{p}^{i} \tau^{\nu}\right) \\
\tilde{x}^{i}=\sqrt{\frac{\alpha^{\prime}}{2}}\left(a_{0}^{i}+a_{0}^{i \dagger}\right), \quad \tilde{p}^{i}=\frac{1}{i \sqrt{2 \alpha^{\prime}}}\left(a_{0}^{i}-a_{0}^{i \dagger}\right),
\end{array}
$$

where $J_{\nu-\frac{1}{2}}$ and $Y_{\nu-\frac{1}{2}}$ are the Bessel functions of the first and second type, respectively. The creation/annihilation operators satisfy the usual oscillator algebra

$$
\left[a_{n}^{i}, a_{m}^{j \dagger}\right]=\left[\tilde{a}_{n}^{i}, \tilde{a}_{m}^{j \dagger}\right]=\delta^{i j} \delta_{n m}, \quad\left[a_{0}^{i}, a_{0}^{j \dagger}\right]=\delta^{i j},
$$


and all the other commutation relations vanish.

The Hamiltonian for the system under consideration can be written as

$$
\begin{array}{r}
H=\frac{1}{\alpha^{\prime} p_{v}}\left(\mathcal{H}_{0}(\tau)+\frac{1}{2} \sum_{n=1}^{\infty} n\left[\Omega_{n}(\tau)\left(a_{n}^{\dagger} \cdot a_{n}+\tilde{a}_{n}^{\dagger} \cdot \tilde{a}_{n}+D\right)-B_{n}(\tau) a_{n} \cdot \tilde{a}_{n}-B_{n}^{*}(\tau) a_{n}^{\dagger} \cdot \tilde{a}_{n}^{\dagger}\right]\right) \\
\Omega_{n}(\tau)=\left(1+\frac{\nu}{4 \tau^{2} n^{2}}\right)|Z|^{2}+|W|^{2}-\frac{\nu}{2 n \tau}\left(Z W^{*}+Z^{*} W\right), \\
B_{n}(\tau)=\left(1+\frac{\nu}{4 \tau^{2} n^{2}}\right) Z^{2}+W^{2}-\frac{\nu}{\tau n} Z W(13)
\end{array}
$$

where $D=\delta^{i i}$. The dot denotes the scalar product in the transverse space and

$$
W(2 n \tau) \equiv e^{-i \frac{\pi}{2} \nu} \sqrt{\pi n \tau}\left[J_{\nu+\frac{1}{2}}(2 n \tau)-i Y_{\nu+\frac{1}{2}}(2 n \tau)\right]
$$

The term $\mathcal{H}_{0}$ in the first line of (13) is the zero-mode part, which is obtained treating the zero mode as a harmonic oscillator with time-dependent frequency [.5]:

$$
\mathcal{H}_{0}(\tau)=\frac{\alpha^{\prime}}{2}\left[\left(p_{0}^{i}\right)^{2}+\frac{k}{4 \alpha^{\prime 2} \tau^{2}}\left(x_{0}^{i}\right)^{2}\right] .
$$

The Hamiltonian, as expressed in terms of the modes $a_{n}, \tilde{a}_{n}, a_{0}$, is non-diagonal. However, in the limit $\tau=-\infty$ the Hamiltonian is diagonal and the metric is flat. In order to diagonalize it in a finite time, a new set of time-dependent string modes is defined as

$$
\begin{array}{ll}
\mathcal{A}_{n}^{i}(\tau)=f_{n}(\tau) a_{n}^{i}+g_{n}^{*}(\tau) \tilde{a}_{n}^{i \dagger}, & \mathcal{A}_{n}^{i \dagger}(\tau)=f_{n}^{*}(\tau) a_{n}^{i \dagger}+g_{n}(\tau) \tilde{a}_{n}^{i}, \\
\tilde{\mathcal{A}}_{n}^{i}(\tau)=f_{n}(\tau) \tilde{a}_{n}^{i}+g_{n}^{*}(\tau) a_{n}^{i \dagger}, & \tilde{\mathcal{A}}_{n}^{i \dagger}(\tau)=f_{n}^{*}(\tau) \tilde{a}_{n}^{i \dagger}+g_{n}(\tau) a_{n}^{i},
\end{array}
$$

where

$$
\begin{aligned}
f_{n}(\tau) & =\frac{1}{2} \sqrt{\frac{w_{n}}{n}} e^{2 i w_{n} \tau}\left[Z(2 n \tau)+\frac{i}{2 w_{n}} \dot{Z}(2 n \tau)\right], \\
g_{n}(\tau) & =\frac{1}{2} \sqrt{\frac{w_{n}}{n}} e^{-2 i w_{n} \tau}\left[Z(2 n \tau)-\frac{i}{2 w_{n}} \dot{Z}(2 n \tau)\right],
\end{aligned}
$$

and

$$
\begin{array}{r}
w_{n}(\tau)=\sqrt{n^{2}+\frac{k}{4 \tau^{2}}}, \\
\dot{Z}(2 n \tau)=\partial_{\tau} Z(2 n \tau)=\frac{\nu}{\tau} Z(2 n \tau)-2 n W(2 n \tau),
\end{array}
$$

with $Z(2 n \tau)$ and $W(2 n \tau)$ defined in (9) and (14), respectively.

We can see that this diagonalization process is in fact a canonical transformation, since

$$
\left|f_{n}(\tau)\right|^{2}-\left|g_{n}(\tau)\right|^{2}=1
$$


and the commutation relations for the new set of modes are

$$
\left[\mathcal{A}_{n}^{i}(\tau), \mathcal{A}_{m}^{j \dagger}(\tau)\right]=\delta_{n m} \delta^{i j}, \quad\left[\tilde{\mathcal{A}}_{n}^{i}(\tau), \tilde{\mathcal{A}}_{m}^{j \dagger}(\tau)\right]=\delta_{n m} \delta^{i j}, \quad\left[\mathcal{A}_{n}^{i}(\tau), \tilde{\mathcal{A}}_{m}^{j \dagger}(\tau)\right]=0
$$

Having established the model and the notation, let's deal with the object of our paper.

\section{DYNAMICAL ENTANGLEMENT AND ENTROPY OPERATOR}

In this section we are going to analyze the model presented above as seen by an observer in the vacuum $\left|0, p_{v}\right\rangle$ at $\tau=-\infty$. The analysis consists of beginning in the vacuum at $\tau=-\infty$ and studying how this state evolves to $\tau=0$. To this end we are going to construct a Bogoliubov transformation, relating the Hilbert space in $\tau=-\infty$ with the Hilbert space at a finite time. Notice that, owing to the symmetry of the metric $\tau \rightarrow-\tau$, this system could also be analyzed from $\tau=+\infty$ evolving back to $\tau=0$. We consider the vacuum $\left|0, p_{v}\right\rangle$ as the flat vacuum annihilated by the $a_{n}^{i}, \tilde{a}_{n}^{i}$ modes and look for the vacuum

$\left|0(\tau), p_{v}\right\rangle$ annihilated by $\mathcal{A}_{n}(\tau)$ and $\tilde{\mathcal{A}}_{n}(\tau)$. We are going to show that this vacuum is a coherent state defined by an entanglement entropy operator.

The first step in our construction is to notice that the expression (16) can be written as the following general $S U(1,1)$ Bogoliubov transformation

$$
\begin{aligned}
\left(\begin{array}{c}
\mathcal{A}_{n}^{i}(\tau) \\
\widetilde{\mathcal{A}}_{n}^{i \dagger}(\tau)
\end{array}\right) & =\mathbb{B}_{n}(\tau)\left(\begin{array}{c}
a_{n}^{i} \\
\widetilde{a}_{n}^{i \dagger}
\end{array}\right), \\
\left(\mathcal{A}_{n}^{i \dagger}(\tau)-\widetilde{\mathcal{A}}_{n}^{i}(\tau)\right) & =\left(a_{n}^{i \dagger}-\widetilde{a}_{n}^{i}\right) \mathbb{B}_{n}^{-1}(\tau),
\end{aligned}
$$

where the $S U(1,1)$ matrix transformation is given by

$$
\mathbb{B}_{n}(\tau)=\left(\begin{array}{cc}
f_{n}(\tau) & g_{n}^{*}(\tau) \\
g_{n}(\tau) & f_{n}^{*}(\tau)
\end{array}\right), \quad\left|f_{n}(\tau)\right|^{2}-\left|g_{n}(\tau)\right|^{2}=1
$$

in such a way that we have a canonical transformation mapping asymptotically flat string operators into operators defined in a finite $\tau$. Now, we are going to find the transformation for the states, in order to have a map between the flat Hilbert space and the finite time one. To this end it is interesting to recognize that the structure of the Bogoliubov transformation (21), (22) and (23) is the same as the general unitary $S U(1,1)$ (TFD) formulation [7, 8, 9]. However, as mentioned in the introduction, here there is no duplication of the degrees of 
freedom by introducing an auxiliary non-physical system and both set of oscillators are dynamical one. We can take advantage of the well-known $\mathrm{SU}(1,1)$ structure cited above to deal with the system in a schematic way. For example, using the polar decomposition for the Bogoliubov matrix elements, (23) can be rewritten as

$$
\mathbb{B}_{n}(\tau)=\frac{e^{s_{n} \tau_{3}}}{\sqrt{1-\bar{f}_{n}}}\left(\begin{array}{cc}
1 & -\bar{f}_{n}^{\alpha_{n}} \\
-\bar{f}_{n}^{1-\alpha_{n}} & 1
\end{array}\right)
$$

where $\tau_{3}$ is a Pauli matrix,

$$
\begin{array}{r}
\bar{f}_{n}=\bar{f}_{n}(\tau)=\frac{\left|g_{n}(\tau)\right|^{2}}{\left|f_{n}(\tau)\right|^{2}}, \quad \alpha_{n}=\alpha_{n}(\tau)=\frac{\ln \left(-\frac{g_{n}^{*}(\tau)}{f_{n}(\tau)}\right)}{\ln \left(\bar{f}_{n}(\tau)\right)}=\frac{1}{2}-i \frac{\left(\phi_{n}(\tau)+\varphi_{n}(\tau)\right)}{\ln \left(\bar{f}_{n}(\tau)\right)}, \\
s_{n}=s_{n}(\tau)=i \varphi_{n}(\tau)=\frac{1}{2} \ln \left(\frac{f_{n}(\tau)}{f_{n}^{*}(\tau)}\right),
\end{array}
$$

and $\alpha_{n}+\alpha_{n}^{*}=1$. Furthermore we can write down a state annihilated by $\mathcal{A}_{n}^{i}(\tau)$ and $\tilde{\mathcal{A}}_{n}^{i}(\tau)$ at each $\tau$, and interpret it as the vacuum of the Bogoliubov transformed system. Such a state has the structure of a condensed state of $a_{n}^{i \dagger} \widetilde{a}_{n}^{i \dagger}$-pair and, by construction, it is also a superposition of $S U(1,1)$ coherent states

$$
\left|0(\tau), p_{v}\right\rangle=\prod_{n=1}^{\infty}\left(1-\bar{f}_{n}\right)^{\alpha_{n} D} e^{\bar{f}_{n}^{\alpha_{n}} a_{n}^{\dagger} \cdot \cdot_{n}^{\dagger}}\left|0, p_{v}\right\rangle,
$$

with its dual state being

$$
\left\langle 0(\tau), p_{v}\right|=\left\langle 0, p_{v}\right| \prod_{n=1}^{\infty}\left(1-\bar{f}_{n}\right)^{\left(1-\alpha_{n}\right) D} e^{\bar{f}_{n}^{1-\alpha_{n}} a_{n} \cdot \widetilde{a}_{n}} .
$$

Since $\bar{f}_{n}^{\alpha_{n}}(\tau)=-\frac{g_{n}^{*}(\tau)}{f_{n}(\tau)}$, one can see that

$$
\mathcal{A}_{n}^{i}(\tau)\left|0(\tau), p_{v}\right\rangle=\tilde{\mathcal{A}}_{n}^{i}(\tau)\left|0(\tau), p_{v}\right\rangle=0
$$

and in fact the condensed state (26) is the vacuum of the system at a finite time. The structure of the state (26) shows us that, owing to this time dependent background, the string is excited in a particular way characterized by a condensed state of left and right moving string modes. Actually, this condensed state is an entangled state of string modes similar to TFD states. However, it is important to stress the crucial difference between the usual TFD and the use of its structure presented here: while in TFD the entanglement is between the original system with an auxiliary one [20], here the Bogoliubov transformation 
entangles the right and left moving string oscillators. Besides, the string entangled state is generated by the background and it is observer dependent.

Again inspired in the TFD, it is possible to show that the entangled state defined in (26) can be obtained from the following expression:

$$
\left|0(\tau), p_{v}\right\rangle=e^{i \Psi(\tau)} e^{-\frac{\mathrm{K}}{2}} e^{\sum_{n} a_{n}^{\dagger} \cdot \widetilde{a}_{n}^{\dagger}}\left|0, p_{v}\right\rangle
$$

where $\Psi(\tau)$ is

$$
\Psi(\tau)=\sum_{n=1}^{\infty}\left[\frac{\left(\phi_{n}(\tau)+\varphi_{n}(\tau)\right)}{\ln \left(\bar{f}_{n}(\tau)\right)} \mathbf{K}_{n}\right]
$$

and $\mathbf{K}$ is

$$
\mathbf{K}=\sum_{n=1}^{\infty} \mathbf{K}_{n}=\sum_{n=1}^{\infty}\left(K_{n}+\widetilde{K}_{n}\right)
$$

with

$$
K_{n}=-\left[a_{n}^{\dagger} \cdot a_{n} \ln \left(\left|g_{n}(\tau)\right|^{2}\right)-a_{n} \cdot a_{n}^{\dagger} \ln \left(\left|f_{n}(\tau)\right|^{2}\right)\right] .
$$

The expression for $\widetilde{K}_{n}$ is obtained changing $a_{n}, a_{n}^{\dagger}$ for $\widetilde{a}_{n}, \widetilde{a}_{n}^{\dagger}$ in $K_{n}$. Now we have a operator mapping $\left|0, p_{v}\right\rangle$ into $\left|0(\tau), p_{v}\right\rangle$. In the TFD approach, the $\mathbf{K}$ is called entropy operator since its expectation value in the thermal vacuum furnishes the thermodynamical entropy of the system. Here it can be viewed as the generator of the entangled state (26) and it is called in this context the entanglement entropy operator [14, 16]. Nevertheless, the structure of the string entanglement generated by the entropy operator differs from the one used in [14, 16] by complex parameters. In fact, we have a more general structure since the entanglement of [14, 16] is related to the usual TFD formulation, while in this work the entropy operator is related to the general unitary $\mathrm{SU}(1,1)$ formulation of TFD. As it is shown in 21, 22], the expectation values do not depend on $\alpha_{n}$ and we do not need to worry about the imaginary part of the exponentials defined in (29). For example, if we consider the $\mathbf{K}$ expectation value in $\left|0(\tau), p_{v}\right\rangle$, we find

$$
\mathcal{S}(\tau)=-2 d \sum_{n=1}^{\infty}\left[\left|g_{n}(\tau)\right|^{2} \ln \left(\left|g_{n}(\tau)\right|^{2}\right)-\left(1+\left|g_{n}(\tau)\right|^{2}\right) \ln \left(1+\left|g_{n}(\tau)\right|^{2}\right)\right]
$$

since $\left\langle 0(\tau), p_{v} \mid 0(\tau), p_{v}\right\rangle=1$ and

$$
\left\langle 0(\tau), p_{v}\left|N_{n}\right| 0(\tau), p_{v}\right\rangle=\left\langle 0(\tau), p_{v}\left|\widetilde{N}_{n}\right| 0(\tau), p_{v}\right\rangle=D\left|g_{n}(\tau)\right|^{2}
$$

for $N_{n}=a_{n}^{\dagger} \cdot a_{n}$ and $\widetilde{N}_{n}=\widetilde{a}_{n}^{\dagger} \cdot \widetilde{a}_{n}$. 
Noticing that (29) can have the alternative form

$$
\left|0(\tau), p_{v}\right\rangle=e^{i \Psi(\tau)} \sum_{n_{k}=0}^{\infty} \prod_{k}\left(\frac{\left|g_{k}(\tau)\right|^{2 n_{k}}}{\left|f_{k}(\tau)\right|^{2 n_{k}+2}}\right)^{D}\left|n_{k}, \widetilde{n}_{k}, p_{v}\right\rangle,
$$

the expectation value of $\mathbf{K}$ can also be written as

$$
\mathcal{S}(\tau)=\sum_{n} \mathcal{W}_{n}(\tau) \ln \mathcal{W}_{n}(\tau)
$$

where

$$
\mathcal{W}_{n}(\tau)=\prod_{k}\left(\frac{\left|g_{k}(\tau)\right|^{2 n_{k}}}{\left|f_{k}(\tau)\right|^{2 n_{k}+2}}\right)^{D},
$$

are the eigenvalues of the system's density matrix.

In order to investigate the entropy's behaviour as $n|\tau| \rightarrow 0$ and $n|\tau| \rightarrow \infty$, note that $\left|g_{n}(\tau)\right|^{2}$ can be expressed in terms of $\Omega_{n}$ and $w_{n}$ given in (13) and (18) respectively:

$$
\left|g_{n}(\tau)\right|^{2}=\frac{1}{4 w_{n}}\left(n \Omega_{n}-2 w_{n}\right) \text {. }
$$

So, it is just needed to know how $\Omega_{n}$ behaves in these two limits. For $n|\tau| \gg 1$, one has [5]

$$
\Omega_{n}(\tau)=2+\frac{k}{4 n^{2} \tau^{2}}-\frac{k^{2}}{64 n^{4} \tau^{4}}+\frac{k^{2}(2+k)}{512 n^{6} \tau^{6}}+\ldots
$$

and, after some cancellations, $\left|g_{n}(\tau)\right|^{2}$ takes the form

$$
\left|g_{n}(\tau)\right|^{2} \cong \frac{k^{2}}{1024 \tau^{6} n^{6}}
$$

Substituting the expression above in (33), the dependence of the entropy with respect to $\tau$ is

$$
\mathcal{S}(\tau) \approx \frac{D k^{2}}{483840 \tau^{6}} \times\left[\pi^{6} \ln \left(\frac{1024 \tau^{6}}{k^{2}}\right)-5670 \zeta^{\prime}(6)\right]
$$

where $\zeta^{\prime}(6)$ is the first derivative of the Zeta function. The result obtained above guarantees that the entropy is positive definite and it increases when goes from $\tau=-\infty$ to 0 . If we take the limit $|\tau| \rightarrow \infty$, the entropy tends to zero, as expected for the asymptotically flat states.

Now we analyze $\mathcal{S}$ close to the singularity $(n|\tau| \ll 1)$. In this case, $\Omega_{n}$ takes the form

$$
\Omega_{n}(\tau) \cong \frac{\pi}{(n \tau)^{2 \nu} \cos ^{2}(\pi \nu)}\left(\frac{1}{\left[\Gamma\left(\frac{1}{2}-\nu\right)\right]^{2}}+\frac{\nu}{4\left[\Gamma\left(\frac{3}{2}-\nu\right)\right]^{2}}+\frac{\nu}{\Gamma\left(\frac{3}{2}-\nu\right) \Gamma\left(\frac{1}{2}-\nu\right)}\right)
$$

or

$$
\Omega_{n} \approx C \times \frac{1}{(n \tau)^{2 \nu}}
$$


where $C$ is the constant part of (42). As $n|\tau| \ll 1, w_{n} \rightarrow \frac{\sqrt{k}}{2|\tau|}$, we have:

$$
\begin{aligned}
\mathcal{S}(\tau)=-2 D \sum_{n=1}^{\infty}\left[\left(\frac{C}{2 \sqrt{k}} \times(n|\tau|)^{1-2 \nu}\right.\right. & \left.-\frac{1}{2}\right) \ln \left(\frac{\frac{C}{2 \sqrt{k}} \times(n|\tau|)^{1-2 \nu}-\frac{1}{2}}{\frac{C}{2 \sqrt{k}} \times(n|\tau|)^{1-2 \nu}+\frac{1}{2}}\right) \\
& \left.-\ln \left(\frac{C}{2 \sqrt{k}} \times(n|\tau|)^{1-2 \nu}+\frac{1}{2}\right)\right] .
\end{aligned}
$$

One can see from the equation above that the entropy diverges when $n|\tau| \ll 1$. This behaviour of $\mathcal{S}(\tau)$ close to the singularity is owing to the creation of high frequency modes. Note that it is possible to avoid the singularity using an analytic continuation of the Bessel functions, although this procedure leads to a discontinuity in the time derivative in the zero

mode sector 5]. However, using this approach, the entropy operator does not change and therefore it still diverges. This means that, for an observer in $\tau=-\infty$, there is a complete loss of information when the string approaches the singularity, and the positive time region turns out to be inaccessible. This is clear if we calculate the following projection

$$
\left\langle p_{v}, 0 \mid 0(\tau), p_{v}\right\rangle=e^{-D \sum_{n} \ln \left(1+\left|g_{n}(\tau)\right|^{2}\right)} e^{2 i D \sum_{n}\left[\left(\phi_{n}(\tau)+\varphi_{n}(\tau)\right) \frac{\ln \left|f_{n}(\tau)\right|^{2}}{\ln \left(\bar{f}_{n}(\tau)\right)}\right]}
$$

and take the limit $n|\tau| \ll 1$. The result is $\left\langle p_{v}, 0 \mid 0(\tau), p_{v}\right\rangle=0$, which shows that in this limit the states at different times are unitarily inequivalent to the vacuum at $\tau=-\infty$. In other words, this result shows that close to the singularity, the system defined by the $2 \mathrm{~d}$ worldsheet quantum field theory is led to another representation of the canonical commutation relations, which is unitarily inequivalent to the representation at $\tau=-\infty$. We will return to this point in the conclusions.

\section{THERMAL EFFECTS}

We have shown that an entanglement entropy appears when the string propagates in this time-dependent geometry. The loss of information related to such a behaviour can be manifested macroscopically and it is natural to search for thermal effects in this context. It can be achieved if we take advantage of the $S U(1,1)$ structure of the system and identify

$$
\sinh ^{2}\left(\theta_{n}\right)=\left|g_{n}(\tau)\right|^{2}, \quad \cosh ^{2}\left(\theta_{n}\right)=\left|f_{n}(\tau)\right|^{2}
$$


where the parameters $\theta_{n}$ encode the information of all the relevant parameters of the theory. Let's define the following potential as a Legendre transformation of the entanglement entropy

$$
\mathcal{F}(\beta, T)=\mathcal{E}(\tau)-T \mathcal{S}(\tau)
$$

where $\mathcal{E}=\left\langle 0(\tau), p_{v}\left|H_{\text {flat }}\right| 0(\tau), p_{v}\right\rangle$ is the expectation value of the asymptotically flat ( $\tau=$ $-\infty)$ Hamiltonian operator, and $T=\partial \mathcal{E} / \partial \mathcal{S}$ is a positive definite parameter. If we look at values of $\theta_{n}$ that make $\mathcal{F}$ stationary and considering negligible the variations of $T$ with respect to time, we have

$$
\left|g_{n}(\tau)\right|^{2}=\sinh ^{2}\left(\theta_{n}\right)=\frac{1}{e^{\frac{1}{T}\left(\frac{n}{\alpha^{\prime} p_{v}}\right)}-1} .
$$

For this configuration of $\left|g_{n}(\tau)\right|^{2}$ the vacuum $\left|0(\tau), p_{v}\right\rangle$ is a maximal entangled state as can be proved following Ref. [11]. Under such a specific configuration that maximizes the entanglement entropy, the expression (48) can be viewed as the Boltzmann distribution for string modes providing that $T$ is identified with the temperature. This scenario can be interpreted as follows: the loss of information's effect, as seen by an asymptotically flat observer, is macroscopically manifested as a heat bath when the string evolves in the time-dependent background.

\section{CONCLUSIONS}

In this work the closed string propagating in a pure dilatonic time dependent background is studied in a new perspective, where the production of entangled states is explored. In Brinkmann coordinates, the evolution of the string states from $\tau=-\infty$ to the singularity at $\tau=0$ is analyzed. From the point of view of an observer in the flat vacuum, the vacuum in a finite time is a $S U(1,1)$ entangled state of right and left moving string modes defined by an entropy operator, which plays the role of the entanglement generator. Its expectation value, evaluated in the finite time vacuum, furnishes the entanglement entropy perceived by the flat observer. In addition, it was shown that close to the singularity the entanglement entropy seen by the observer in flat vacuum diverges. Also, we showed that the Hilbert space close to the singularity is unitarily inequivalent to the flat Hilbert space. These results imply a complete loss of information at the singularity and consequently the positive time region is inaccessible to an observer in $\tau=-\infty$. 
The unitary nonequivalence between the Hilbert spaces is a typical result of dissipative motion, where the entropy operator drives the system through unitarily inequivalent states and it is interpreted as a time evolution controller, as shown in [14, 15]. At this point it is interesting to remember that in Rosen coordinates the string's equation of motion resembles a damped harmonic oscillator equation. So, it is not surprising to find a dissipative behaviour in other coordinate system. Consequently, it is tempting to conjecture that the string entropy operator generates the time evolution of the system. However, there is a crucial difference between the results of [14, 15] and our work. While in [14, 15] the condensed state generated by the entropy operator is also generated by a $\mathrm{SU}(1,1)$ Hamiltonian, in our work the string condensed state is generated by a Bogoliubov transformation and we only have a map between Hilbert spaces, which turns out to be non unitary close to the singularity. Although the string Hamiltonian (13) also has a $\mathrm{SU}(1,1)$ structure, we are not able, at present time, to conclude that the string entropy operator generates the evolution of the system. This issue is now under investigation.

At the end it is shown how the loss of information can be macroscopically manifested as a heat bath. By means of a Legendre transformation of the entropy operator, we find a particular stationary configuration which maximizes the entanglement. Besides, this configuration allows us to introduce a parameter that can be interpreted as the temperature, and the expectation value of the number operator at finite time acquires the form of a Boltzmann distribution. So, in this configuration, for asymptotically flat observers, all the information of the string states in a finite time is encoded as a heat bath.

There are many possible extensions of this work. As there is a complete loss of information at the singularity, the approach used in this letter could provide tools to explore the prebig bang phase scenario from a different perspective, where the pre-big-bang states may appear as a heat bath. On the other hand, it will be interesting to see how the string entropy operator used here can appear in the context of reference [23], where it was given an entanglement interpretation for black hole entropy in string theory, using dual field theories. An entropy operator was also used to study information loss in classical dissipative system, in connection with the t'Hooft deterministic quantum mechanics [24, 25]. We can further develop their techniques to investigate this point in the string model studied here, since we have shown that a $2 \mathrm{~d}$ string entropy operator appears naturally owing to time dependent geometry. 


\section{Acknowledgments}

The authors would like to thank Marcelo Botta Cantcheff and M. C. B. Abdalla for useful discussions and FAPESP (Fundação de Amparo à Pesquisa do Estado de São Paulo) for financial support. This paper is dedicated to Marina Gadelha.

[1] D. Amati and C. Klimcik, "Strings in a shock wave background and generation of curved geometry from flat space string theory", Phys. Lett. B 210 (1988) 92.

[2] G. T. Horowitz and A. R. Steif, "Strings in strong gravitational fields", Phys. Rev. D 42 (1990) 1950; "Space-time singularities in string theory", Phys. Rev. Lett. 64 (1990) 260.

[3] R. Brooks, "Plane wave gravitons, curvature singularities and string physics", Mod. Phys. Lett. A 6 (1991) 841.

[4] H. J. de Vega, M. Ramon Medrano and N. Sanchez, "Classical and quantum strings near space-time singularities: gravitational plane waves with arbitrary polarization", Class. Quant. Grav. 10 (1993) 2007.

[5] G. Papadopoulos, J. G. Russo and A. A. Tseytlin, "Solvable model of strings in a time-dependent plane-wave background", Class. Quant. Grav. 20 (2003) 969 arXiv:hep-th/0211289.

[6] M. Gasperini and G. Veneziano, "The pre-big bang scenario in string cosmology", Phys. Rept. 373 (2003) 1 arXiv:hep-th/0207130.

[7] M. C. B. Abdalla and A. L. Gadelha, "General unitary SU(1,1) TFD formulation", Phys. Lett. A 322 (2004) 31 arXiv:hep-th/0309254.

[8] M. C. B. Abdalla, A. L. Gadelha and D. L. Nedel, "On the entropy operator for the general SU(1,1) TFD formulation", Phys. Lett. A 334 (2005) 123 arXiv:hep-th/0409116.

[9] M. C. B. Abdalla, A. L. Gadelha and I. V. Vancea, "On the SU(1,1) thermal group of bosonic strings and D-branes", Phys. Rev. D 66 (2002) 065005 arXiv:hep-th/0203222.

[10] M. C. B. Abdalla, A. L. Gadelha and D. L. Nedel, "General unitary TFD formulation for superstrings", Proc. Sci. WC2004 (2004) 032 arXiv:hep-th/0412128.

[11] A. E. Santana, F. C. Khanna, M. Revzen, "Entropy of entangled states and SU(1,1) and SU(2) symmetries", Phys. Rev. A 65 (2002) 032119. 
[12] S. De Filippo and G. Vitiello, "Vacuum structure for unstable particles," Lett. Nuovo Cim. 19 (1977) 92.

[13] P. Garbaczewski and G. Vitiello, "A canonical description of the solitary quantum decay", Nuovo Cim. A 44 (1978) 108.

[14] E. Celeghini, M. Rasetti and G. Vitiello, "Quantum dissipation," Annals Phys. 215 (1992) 156.

[15] E. Alfinito, R. Manka and G. Vitiello, "Double universe", arXiv:hep-th/9705134.

[16] A. Iorio, G. Lambiase and G. Vitiello, "Entangled quantum fields near the event horizon and entropy," Annals Phys. 309 (2004) 151.

[17] B. Chen, Y. l. He and P. Zhang, "Exactly solvable model of superstring in plane-wave background with linear null dilaton," Nucl. Phys. B 741 (2006) 269 arXiv:hep-th/0509113.

[18] S. R. Das and J. Michelson, "pp wave big bangs: Matrix strings and shrinking fuzzy spheres," Phys. Rev. D 72 (2005) 086005 arXiv:hep-th/0508068.

[19] S. R. Das and J. Michelson, "Matrix membrane big bangs and D-brane production," arXiv:hep-th/0602099

[20] Y. Takahasi and H. Umezawa, "Thermo Field Dynamics", Collect. Phenom. 2 (1975) 55.

[21] H. Umezawa, Advanced Field Theory: Micro, Macro and Thermal Field, American Institute of Physics Press, 1995.

[22] P. A. Henning, "Thermo field dynamics for quantum fields with continuous mass spectrum", Phys. Rept. 253 (1995) 235.

[23] R. Brustein, M. B. Einhorn and A. Yarom, "Entanglement interpretation of black hole entropy in string theory," JHEP 0601 (2006) 098 arXiv:hep-th/0508217.

[24] M. Blasone, P. Jizba and G. Vitiello, "Dissipation and quantization", Phys. Lett. A 287 (2001) 205 arXiv:hep-th/0007138.

[25] G. 't Hooft, "Quantum gravity as a dissipative deterministic system", Class. Quant. Grav. 16 (1999) 3263 arXiv:gr-qc/9903084; G. 't Hooft, "Determinism and dissipation in quantum gravity", arXiv:hep-th/0003005. 